\title{
Group Encryption: Non-interactive Realization in the Standard Model
}

\author{
Julien Cathalo ${ }^{1, \star}$, Benoît Libert ${ }^{1, \star \star}$, and Moti Yung ${ }^{2}$ \\ 1 Université catholique de Louvain, Crypto Group (Belgium) \\ ${ }^{2}$ Google Inc. and Columbia University (USA)
}

\begin{abstract}
Group encryption (GE) schemes, introduced at Asiacrypt'07, are an encryption analogue of group signatures with a number of interesting applications. They allow a sender to encrypt a message (in the CCA2 security sense) for some member of a PKI group concealing that member's identity (in a CCA2 security sense, as well); the sender is able to convince a verifier that, among other things, the ciphertext is valid and some anonymous certified group member will be able to decrypt the message. As in group signatures, an opening authority has the power of pinning down the receiver's identity. The initial GE construction uses interactive proofs as part of the design (which can be made non-interactive using the random oracle model) and the design of a fully non-interactive group encryption system is still an open problem. In this paper, we give the first GE scheme, which is a pure encryption scheme in the standard model, i.e., a scheme where the ciphertext is a single message and proofs are non-interactive (and do not employ the random oracle heuristic). As a building block, we use a new public key certification scheme which incurs the smallest amount of interaction, as well.
\end{abstract}

Keywords: Group encryption, anonymity, provable security.

\section{Introduction}

Group encryption (GE) schemes, introduced by Kiayias, Tsiounis and Yung [29], are the encryption analogue of group signatures [16. The latter primitives basically allow a group member to sign messages in the name of a group without revealing his identity. In a similar spirit, GE systems aim to hide the identity of a ciphertext's recipient and still guarantee that he belongs to a population of registered members in a group administered by a group manager (GM). A sender can generate an anonymous encryption of some plaintext $m$ intended for a receiver holding a public key that was certified by the GM (message security and receiver anonymity being both in the CCA2 sense). The ciphertext is prepared while leaving an opening authority (OA) the ability to "open" the ciphertext

\footnotetext{
* This author's research was supported by the Belgian Walloon Region project ALAWN (Programme Wist 2).

** This author acknowledges the Belgian National Fund for Scientific Research (F.R.S.F.N.R.S.) for their financial support.
} 
(analogously to the opening operation in group signatures) and uncover the receiver's name. At the same time, the sender should be able to convince a verifier that (1) the ciphertext is a valid encryption under the public key of some group member holding a valid certificate; (2) if necessary, the opening authority will be able to find out who the receiver is; (3) (optionally) the plaintext is a witness satisfying some public relation.

Motivations. The GE primitive was motivated by various privacy applications such as anonymous trusted third parties or oblivious retriever storage. Many cryptographic protocols such as fair exchange, fair encryption or escrow encryption, involve trusted third parties that remain offline most of the time and are only involved to resolve problems. Group encryption allows one to verifiably encrypt some message to such a trusted third party while hiding his identity among a set of possible trustees. For instance, a user can encrypt a key (e.g., in an "international key escrow system") to his own national trusted representative without letting the ciphertext reveal the latter's identity, which could leak information on the user's citizenship. At the same time, everyone can be convinced that the ciphertext is heading for an authorized trustee.

Group encryption also finds applications in ubiquitous computing, where anonymous credentials must be transferred between peer devices belonging to the same group. Asynchronous transfers may require to involve an untrusted storage server to temporarily store encrypted credentials. In such a situation, GE schemes may be used to simultaneously guarantee that (1) the server retains properly encrypted valid credentials that it cannot read; (2) credentials have a legitimate anonymous retriever; (3) if necessary, an authority will be able to determine who the retriever is.

By combining cascaded group encryptions using multiple trustees and according to a sequence of identity discoveries and transfers, one can also implement group signatures where signers can flexibly specify how a set of trustees should operate to open their signatures.

Prior Works. Kiayias, Tsiounis and Yung (KTY) [29] formalized the concept of group encryption and provided a suitable security modeling. They presented a modular design of GE system and proved that, beyond zero-knowledge proofs, anonymous public key encryption schemes with CCA2 security, digital signatures, and equivocal commitments are necessary to realize the primitive. They also showed how to efficiently instantiate their general construction using Paillier's cryptosystem 35. (or, more precisely, a modification of the CamenischShoup [13] variant of Paillier). While efficient, their scheme is not a single message encryption, since it requires the sender to interact with the verifier in a $\Sigma$-protocol to convince him that the aforementioned properties are satisfied. Interaction can be removed using the Fiat-Shamir paradigm [20] (and thus the random oracle model [4]), but only heuristic arguments [22] (see also [14]) are then possible in terms of security.

Independently, Qin et al. 36] considered a closely related primitive with noninteractive proofs and short ciphertexts. However, they avoid interaction by 
explicitly employing a random oracle and also rely on strong interactive assumptions. As we can see, none of these schemes is a truly non-interactive encryption scheme without the random oracle idealization.

Our Contribution. As already noted in various contexts such as anonymous credentials [2], rounds of interaction are expensive and even impossible at times as, in some applications, proofs should be verifiable by third parties that are not present when provers are available. In the setting of group encryption, this last concern is even more constraining as it requires the sender, who may be required to repeat proofs with many verifiers, to maintain a state and remember the random coins that he uses to encrypt every single ciphertext. In the frequent situation where many encryptions have to be generated using independent random coins, this becomes a definite bottleneck.

This paper solves the above problems and describes the first realization of group encryption which is a fully non-interactive encryption scheme with CCA2security and anonymity in the standard model. In our scheme, senders do not need to maintain a state: thanks to the Groth-Sahai [27] non-interactive proof systems, the proof of a ciphertext can be generated once-and-for-all at the same time as the ciphertext itself. Furthermore, using suitable parameters and for a comparable security level, we can also shorten ciphertexts by a factor of 2 in comparison with the KTY scheme. As far as communication goes, the size of proofs allows decreasing by more than $75 \%$ the number of transmitted bits between the sender and the verifier.

Since our goal is to avoid interaction, we also design a joining protocol (i.e., a protocol whereby the user effectively becomes a group member and gets his public key certified by the GM) which requires the smallest amount of interaction: as in the Kiayias-Yung group signature [30, only two messages have to be exchanged between the GM and the user and the latter need not to prove anything about his public key. In particular, rewinding is not necessary in security proofs and the join protocol can be safely executed in a concurrent environment, when many users want to register at the same time. The join protocol uses a noninteractive public key certification scheme where discrete-logarithm-type public keys can be signed as if they were ordinary messages (and without knowing the matching private key) while leaving the ability to efficiently prove knowledge of the certificate/public key using the Groth-Sahai techniques. To certify users without having to rewind 1 in security proofs, the KTY scheme uses groups of hidden order (and more precisely, Camenisch-Lysyanskaya signatures [12]). In public order groups, to the best of our knowledge, our construction is the first certification method that does not require any form of proof of knowledge of private keys. We believe it to be of independent interest as it can be used to construct group signatures (in the standard model) where the joining mechanism tolerates concurrency in the model of [30. without demanding more than two moves of interaction.

${ }^{1}$ Although the simulator does not need to rewind proofs of knowledge in [29], users still have to interactively prove the validity of their public key. 
OrGanizATION. In section 2, we describe the intractability assumptions that we need and recall the KTY model of group encryption. Section 3 explains the building blocks of our construction and notably describes our certification scheme. Our GE system is depicted in section 4.

\section{Background}

In the paper, when $S$ is a set, $x \stackrel{\$}{\leftarrow} S$ denotes the action of choosing $x$ at random in $S$. By $a \in \operatorname{poly}(\lambda)$, we mean that $a$ is a polynomial in $\lambda$ while $b \in \operatorname{negl}(\lambda)$ says that $b$ is a negligible function of $\lambda$. When $a$ and $b$ are two binary strings, $a \| b$ stands for their concatenation.

\subsection{Complexity Assumptions}

We use groups $\left(\mathbb{G}, \mathbb{G}_{T}\right)$ of prime order $p$ with an efficiently computable map $e: \mathbb{G} \times \mathbb{G} \rightarrow \mathbb{G}_{T}$ such that $e\left(g^{a}, h^{b}\right)=e(g, h)^{a b}$ for any $(g, h) \in \mathbb{G} \times \mathbb{G}, a, b \in \mathbb{Z}$ and $e(g, h) \neq 1_{\mathbb{G}_{T}}$ whenever $g, h \neq 1_{\mathbb{G}}$.

In this setting, we rely on an assumption introduced in [7] that allows constructing efficient non-interactive proofs as pointed out in 27.

Definition 1. The Decision Linear Problem (DLIN) in $\mathbb{G}$, is to distinguish the distribution $D_{1}=\left\{\left(g, g^{a}, g^{b}, g^{a c}, g^{b d}, g^{c+d}\right) \mid a, b, c, d \stackrel{\$}{\leftarrow} \mathbb{Z}_{p}^{*}\right\}$ from the distribution $D_{2}=\left\{\left(g, g^{a}, g^{b}, g^{a c}, g^{b d}, g^{z}\right) \mid a, b, c, d, z \stackrel{\$}{\leftarrow} \mathbb{Z}_{p}^{*}\right\}$. The Decision Linear Assumption is the intractability of DLIN for any PPT algorithm $\mathcal{D}$.

This problem amounts to deciding whether vectors $\overrightarrow{g_{1}}=\left(g^{a}, 1, g\right), \overrightarrow{g_{2}}=\left(1, g^{b}, g\right)$ and $\overrightarrow{g_{3}}$ are linearly dependent or not. We also consider a related computational problem which bears similarities with simultaneous pairing problems [26 25].

Definition 2. The Simultaneous Double Pairing problem (S2P) in $\mathbb{G}$ is, given $\left(g_{1}, g_{2}, g_{1, c}, g_{2, d}\right) \in \mathbb{G}^{4}$, to find a triple $(u, v, w) \in \mathbb{G}^{3} \backslash\left\{\left(1_{\mathbb{G}}, 1_{\mathbb{G}}, 1_{\mathbb{G}}\right)\right\}$ such that $e\left(g_{1}, u\right)=e\left(g_{1, c}, w\right)$ and $e\left(g_{2}, v\right)=e\left(g_{2, d}, w\right)$.

Like the simultaneous triple pairing assumption [25], the hardness of this problem is implied by the DLIN assumption: given $\left(g, g_{1}, g_{2}, g_{1}^{c}, g_{2}^{d}, \eta \stackrel{?}{=} g^{c+d}\right)$ any algorithm that, on input of $\left(g_{1}, g_{2}, g_{1}^{c}, g_{2}^{d}\right)$, outputs a non-trivial $(u, v, w)$ such that $e\left(g_{1}, u\right)=e\left(g_{1}^{c}, w\right), e\left(g_{2}, v\right)=e\left(g_{2}^{d}, w\right)$ allows telling whether $\eta=g^{c+d}$ by testing if $e(g, u \cdot v)=e(\eta, w)$ (since $u=w^{c}$ and $\left.v=w^{d}\right)$.

We also use the Hidden Strong Diffie-Hellman (HSDH) assumption introduced in [10] as a strengthening of the Strong Diffie-Hellman assumption [6].

Definition 3. The $\ell$-Hidden Strong Diffie-Hellman problem $(\ell-H S D H)$ in $\mathbb{G}$ is, given $\left(g, \Omega=g^{\omega}, u\right) \stackrel{\$}{\leftarrow} \mathbb{G}^{3}$ and triples $\left(g^{1 /\left(\omega+s_{i}\right)}, g^{c_{i}}, u^{c_{i}}\right)$ with $c_{1}, \ldots, c_{\ell} \stackrel{\$}{\leftarrow}$ $\mathbb{Z}_{p}^{*}$, to find another triple $\left(g^{1 /(\omega+c)}, g^{c}, u^{c}\right)$ such that $c \neq c_{i}$ for $i=1, \ldots, \ell$.

We finally need the following variant of the Diffie-Hellman assumption.

Definition 4. The Flexible Diffie-Hellman problem (FlexDH) is, given $\left(g, g^{a}, g^{b}\right) \in \mathbb{G}^{3}$, where $a, b \stackrel{\$}{\leftarrow} \mathbb{Z}_{p}^{*}$, to find a triple $\left(C, C^{a}, C^{a b}\right)$ such that $C \neq 1_{\mathbb{G}}$. 
A potentially easier problem considered in [33] only requires to output $\left(C, C^{a b}\right)$ on input of the same values. The latter problem was proved generically hard in prime order groups 33 . In bilinear groups, any algorithm solving either of these two problems would make it easy to recognize $g^{a b c}$ on input of $\left(g, g^{a}, g^{b}, g^{c}\right)$, which is a problem suggested for the first time in [8, Section 8].

\subsection{Model and Security Notions}

Group encryption schemes involve a sender, a verifier, a group manager (GM) that manages the group of receivers and an opening authority (OA) that is able to uncover the identity of ciphertext receivers. A group encryption system is formally specified by the description of a relation $\mathcal{R}$ as well as a collection $\mathrm{GE}=\left(\mathrm{SETUP}, \mathrm{JOIN},\left\langle\mathcal{G}_{r}, \mathcal{R}\right.\right.$, sample s $\left._{\mathcal{R}}\right\rangle$, ENC, DEC, OPEN, $\left.\langle\mathcal{P}, \mathcal{V}\rangle\right)$ of algorithms or protocols. Among these, SETUP is a set of initialization procedures that all take (explicitly or implicitly) a security parameter $\lambda$ as input. They can be split into one that generates a set of public parameters param (a common reference string), one for the GM and another one for the OA. We call them $\operatorname{SETUP}_{\text {init }}(\lambda)$, SETUP $_{\text {GM }}$ (param) and SETUP $P_{\text {AA }}($ param), respectively. The latter two procedures are used to produce key pairs $\left(\mathrm{pk}_{\mathrm{GM}}, \mathrm{sk}_{\mathrm{GM}}\right),\left(\mathrm{pk}_{\mathrm{OA}}\right.$, sk $\left.\mathrm{OA}_{\mathrm{A}}\right)$ for the GM and the OA. In the following, param is incorporated in the inputs of all algorithms although we sometimes omit to explicitly write it.

JOIN $=\left(\mathrm{J}_{\text {user }}, \mathrm{J}_{\mathrm{GM}}\right)$ is an interactive protocol between the GM and the prospective user. As in [30], we will restrict this protocol to have minimal interaction and consist of only two messages: the first one is the user's public key pk sent by Juser to $J_{\mathrm{GM}}$ and the latter's response is a certificate cert $_{\mathrm{pk}}$ for $\mathrm{pk}$ that makes the user's group membership effective. We do not require the user to prove knowledge of his private key sk or anything else about it. In our construction, valid keys will be publicly recognizable and users do not need to prove their validity. After the execution of JOIN, the GM stores the public key pk and its certificate cert pk $_{\text {in }}$ a public directory database.

Algorithm sample allows sampling pairs $(x, w) \in \mathcal{R}$ (made of a public value $x$ and a witness $w$ ) using keys $\left(\mathrm{pk}_{\mathcal{R}}, \mathrm{sk}_{\mathcal{R}}\right)$ produced by $\mathcal{G}_{r}$. Depending on the relation, $\mathbf{s k}_{\mathcal{R}}$ may be the empty string (as will be the case in our scheme). The testing procedure $\mathcal{R}(x, w)$ returns 1 whenever $(x, w) \in \mathcal{R}$. To encrypt a witness $w$ such that $(x, w) \in \mathcal{R}$ for some public $x$, the sender fetches the pair (pk, cert $\left._{\mathrm{pk}}\right)$ from database and runs the randomized encryption algorithm. The latter takes as input $w$, a label $L$, the receiver's pair ( $\mathrm{pk}$, cert $\mathrm{pk}$ ) as well as public keys $\mathrm{pk}_{\mathrm{GM}}$ and $\mathrm{pk}_{\mathrm{OA}}$. Its output is a ciphertext $\psi \leftarrow \mathrm{ENC}\left(\mathrm{pk}_{\mathrm{GM}}, \mathrm{pk}_{\mathrm{OA}}, \mathrm{pk}, \mathrm{cert}_{\mathrm{pk}}, w, L\right)$. On input of the same elements, the certificate cert $_{\mathrm{pk}}$, the ciphertext $\psi$ and the random coins coins $_{\psi}$ that were used to produce it, the non-interactive algorithm $\mathcal{P}$ generates a proof $\pi_{\psi}$ that there exists a certified receiver whose public key was registered in database and that is able to decrypt $\psi$ and obtain a witness $w$ such that $(x, w) \in \mathcal{R}$. The verification algorithm $\mathcal{V}$ takes as input $\psi, \mathrm{pk}_{\mathrm{GM}}, \mathrm{pk}_{\mathrm{OA}}$, $\pi_{\psi}$ and the description of $\mathcal{R}$ and outputs 0 or 1 . Given $\psi, L$ and the receiver's private key sk, the output of DEC is either a witness $w$ such that $(x, w) \in \mathcal{R}$ or a rejection symbol $\perp$. Finally, OPEN takes as input a ciphertext/label pair $(\psi, L)$ and the OA's secret key skoA and returns a receiver's public key pk. 
The security model considers four properties termed correctness, message security, anonymity and soundness. In the following, we sometimes denote by $\left\langle\right.$ output $\left._{A}\right|$ output $\left._{B}\right\rangle \leftarrow\left\langle A\right.$ (input $\left._{A}\right), B$ (input $\left.\left._{B}\right)\right\rangle$ (common-input) the execution of a protocol between $A$ and $B$ obtaining their own outputs from their inputs.

CoRRECTNESS. The correctness property requires that the following experiment returns 1 with overwhelming probability.

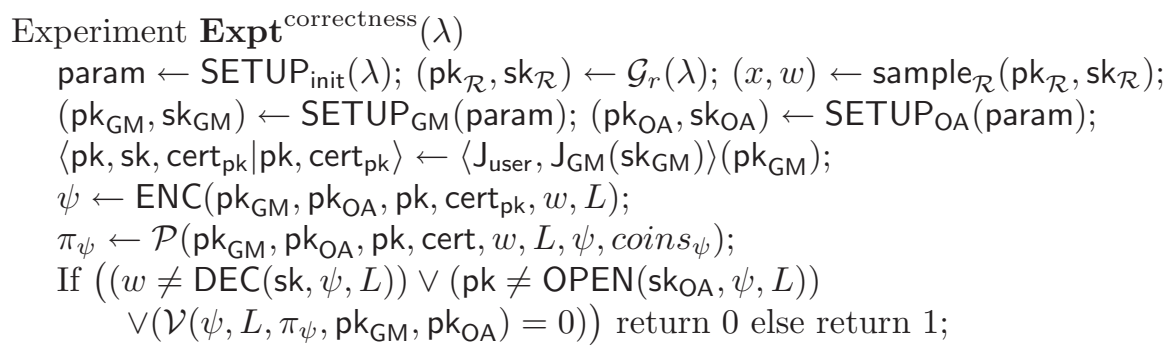

Message SECURITY. The message secrecy property is defined by an experiment where the adversary has access to oracles that may be stateful (and maintain a state across queries) or stateless:

- DEC(sk): is a stateless oracle for the user decryption function DEC. When this oracle is restricted not to decrypt a ciphertext-label pair $(\psi, L)$, we denote it by $\operatorname{DEC} \neg\langle\psi, L\rangle$.

- $\mathrm{CH}_{\text {ror }}^{b}(\lambda, \mathrm{pk}, w, L)$ : is a real-or-random challenge oracle that is only queried once. It returns $\left(\psi, \operatorname{coins}_{\psi}\right)$ such that $\psi \leftarrow \operatorname{ENC}\left(\mathrm{pk}_{\mathrm{GM}}, \mathrm{pk}_{\mathrm{OA}}, \mathrm{pk}, \operatorname{cert}_{\mathrm{pk}}, w, L\right)$ if $b=1$ whereas, if $b=0, \psi \leftarrow \mathrm{ENC}\left(\mathrm{pk}_{\mathrm{GM}}, \mathrm{pk}_{\mathrm{OA}}, \mathrm{pk}, \operatorname{cert}_{\mathrm{pk}}, w^{\prime}, L\right)$ encrypts a random plaintext uniformly chosen in the space of plaintexts of length $O(\lambda)$. In either case, coins $_{\psi}$ are the random coins used to generate $\psi$.

- $\operatorname{PROVE}_{\mathcal{P}, \mathcal{P}^{\prime}}^{b}\left(\mathrm{pk}_{\mathrm{GM}}, \mathrm{pk}_{\mathrm{OA}}, \mathrm{pk}, \operatorname{cert}_{\mathrm{pk}}, \mathrm{pk}_{\mathcal{R}}, x, w, \psi, L\right.$, coins $\left._{\psi}\right)$ : is a stateful oracle that the adversary can query on multiple occasions. If $b=1$, it runs the real prover $\mathcal{P}$ on the inputs to produce an actual proof $\pi_{\psi}$. If $b=0$, the oracle runs a simulator $\mathcal{P}^{\prime}$ that uses the same inputs as $\mathcal{P}$ except witness $w, \operatorname{coins}_{\psi}$ and generates a simulated proof.

These oracles are used in an experiment where the adversary controls the GM, the OA and all members but the honest receiver. The adversary $\mathcal{A}$ is the dishonest GM that certifies the honest receiver in an execution of JOIN. She has oracle access to the decryption function DEC of that receiver. At the challenge phase, she probes the challenge oracle for a label and a pair $(x, w) \in \mathcal{R}$ of her choice. After the challenge phase, she can also invoke the PROVE oracle on multiple occasions and eventually aims to guess the bit $b$ chosen by the challenger.

As pointed out in 29, designing an efficient simulator $\mathcal{P}^{\prime}$ (for executing PROVE $_{\mathcal{P}, \mathcal{P}^{\prime}}^{b}($.$) when b=0$ ) is part of the security proof and might require a simulated common reference string.

Definition 5. A GE scheme satisfies message security if, for any PPT adversary $\mathcal{A}$, the experiment below returns 1 with probability at most $1 / 2+\operatorname{negl}(\lambda)$. 


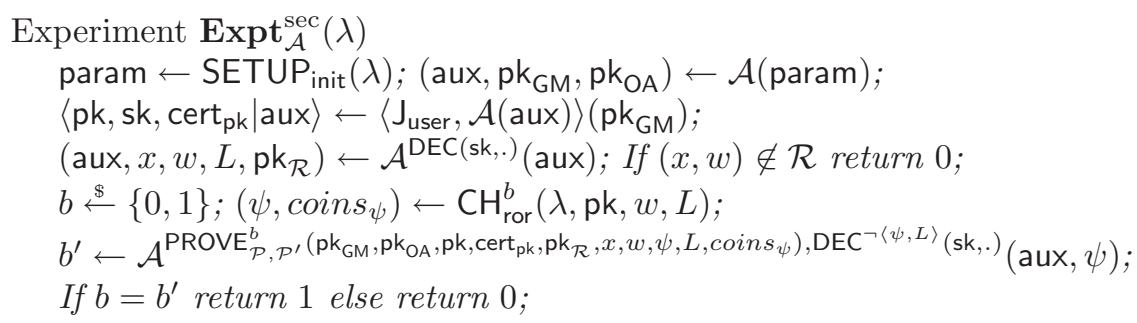

ANONYMITY. In anonymity attacks, the adversary controls the whole system but the opening authority and performs a kind of chosen-ciphertext attack on the encryption scheme of the OA. She registers two keys $\mathrm{pk}_{0}, \mathrm{pk}_{1}$ in database and, for a pair $(x, w) \in \mathcal{R}$ of her choosing, obtains an encryption of $w$ under $\mathrm{pk}_{b}$ for some $b \in\{0,1\}$ chosen by the challenger. She is granted access to decryption oracles w.r.t. both keys $\mathrm{pk}_{0}, \mathrm{pk}_{1}$. In addition, she may invoke the following oracles:

- $\mathrm{CH}_{\mathrm{anon}}^{b}\left(\mathrm{pk}_{\mathrm{GM}}, \mathrm{pk}_{\mathrm{OA}}, \mathrm{pk}_{0}, \mathrm{pk}_{1}, w, L\right)$ : is a challenge oracle that is only queried once by the adversary. It returns a pair $\left(\psi\right.$, coins $\left._{\psi}\right)$ consisting of a ciphertext $\psi \leftarrow \mathrm{ENC}\left(\mathrm{pk}_{\mathrm{GM}}, \mathrm{pk}_{\mathrm{OA}}, \mathrm{pk}_{b}, \operatorname{cert}_{\mathrm{pk}_{b}}, w, L\right)$ and the coin tosses coins $\psi$ that were used to generate $\psi$.

- USER $\left(\mathrm{pk}_{\mathrm{GM}}\right)$ : is a stateful oracle simulating two executions of $J_{\text {user }}$ to introduce two honest users in the group. It uses a string keys where the outputs of the two executions are written.

- OPEN(skoA, .): is a stateless oracle that simulates the opening algorithm on behalf of the OA and, on input of a GE ciphertext, returns the receiver's public key.

Definition 6. A GE scheme satisfies anonymity if, for any PPT adversary $\mathcal{A}$, the experiment below returns 1 with a probability not exceeding $1 / 2+\operatorname{negl}(\lambda)$.

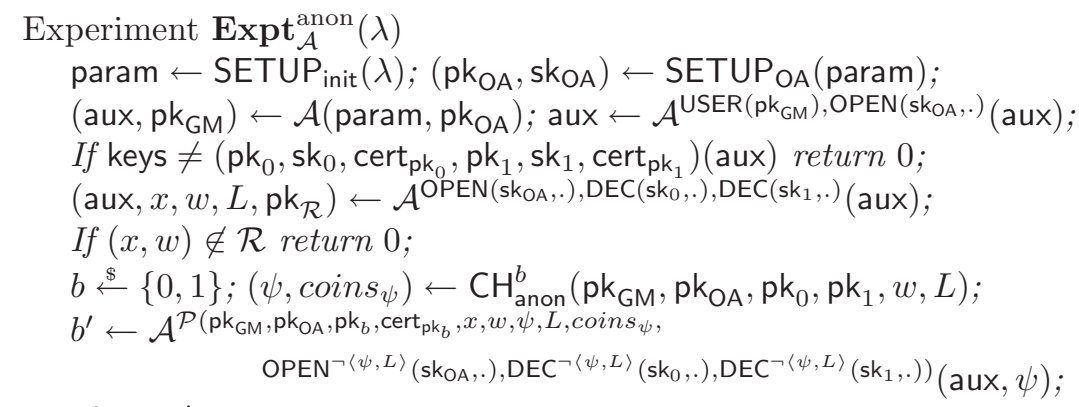

If $b=b^{\prime}$ return 1 else return 0 ;

As shown in 29], GE schemes satisfying the above notion necessarily subsume a key-private (a.k.a. receiver anonymous) 3128 cryptosystem.

SounDNESS. In a soundness attack, the adversary creates the group of receivers by interacting with the honest GM. Her goal is to produce a ciphertext $\psi$ and a convincing proof that $\psi$ is valid w.r.t. a relation $\mathcal{R}$ of her choice but either (1) the opening reveals a receiver's public key pk that does not belong to any group member; (2) the output pk of OPEN is not a valid public key (i.e., pk $\notin \mathcal{P K}$, 
where $\mathcal{P} \mathcal{K}$ is the space of valid public keys); (3) the ciphertext $C$ is not in the

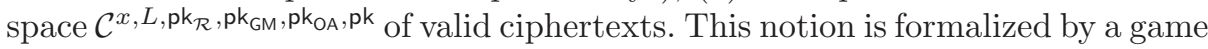
where the adversary is given access to a user registration oracle $\operatorname{REG}\left(\mathrm{sk}_{\mathrm{GM}},.\right)$ that simulates $\mathrm{J}_{\mathrm{GM}}$. This oracle maintains a repository database where registered public keys and their certificates are stored.

Definition 7. A GE scheme is sound if, for any PPT adversary $\mathcal{A}$, the experiment below returns 1 with negligible probability.

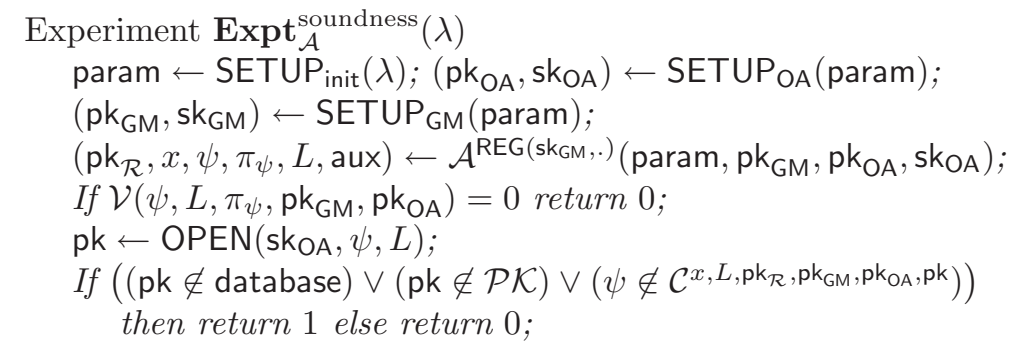

\subsection{Groth-Sahai Proof Systems}

In the following notations, for equal-dimension vectors $\vec{A}$ and $\vec{B}$ containing group elements, $\vec{A} \odot \vec{B}$ stands for their component-wise product.

When based on the DLIN assumption, the Groth-Sahai (GS) proof systems [27] use a common reference string comprising vectors $\overrightarrow{g_{1}}, \overrightarrow{g_{2}}, \overrightarrow{g_{3}} \in \mathbb{G}^{3}$, where $\overrightarrow{g_{1}}=\left(g_{1}, 1, g\right), \overrightarrow{g_{2}}=\left(1, g_{2}, g\right)$ for some $g_{1}, g_{2} \in \mathbb{G}$. To commit to $X \in \mathbb{G}$, one sets $\vec{C}=(1,1, X) \odot{\overrightarrow{g_{1}}}^{r} \odot{\overrightarrow{g_{2}}}^{s} \odot{\overrightarrow{g_{3}}}^{t}$ with $r, s, t \stackrel{\$}{\leftarrow} \mathbb{Z}_{p}^{*}$. When the proof system is configured to give perfectly sound proofs, $\overrightarrow{g_{3}}$ is chosen as $\overrightarrow{g_{3}}=\overrightarrow{g_{1}} \xi_{1} \odot \overrightarrow{g_{2}} \xi_{2}$ with $\xi_{1}, \xi_{2} \stackrel{\$}{\leftarrow} \mathbb{Z}_{p}^{*}$. Commitments $\vec{C}=\left(g_{1}^{r+\xi_{1} t}, g_{2}^{s+\xi_{2} t}, X \cdot g^{r+s+t\left(\xi_{1}+\xi_{2}\right)}\right)$ are then BonehBoyen-Shacham (BBS) ciphertexts that can be decrypted using $\alpha_{1}=\log _{g}\left(g_{1}\right)$, $\alpha_{2}=\log _{g}\left(g_{2}\right)$. In the witness indistinguishability (WI) setting, vectors $\overrightarrow{g_{1}}, \overrightarrow{g_{2}}, \overrightarrow{g_{3}}$ are linearly independent and $\vec{C}$ is a perfectly hiding commitment. Under the DLIN assumption, the two kinds of CRS are indistinguishable.

To commit to an exponent $x \in \mathbb{Z}_{p}$, one computes $\vec{C}=\vec{\varphi}^{x} \odot{\overrightarrow{g_{1}}}^{r} \odot{\overrightarrow{g_{2}}}^{s}$, with $r, s \stackrel{\$}{\leftarrow} \mathbb{Z}_{p}^{*}$, using a CRS comprising vectors $\vec{\varphi}, \overrightarrow{g_{1}}, \overrightarrow{g_{2}}$. In the soundness setting $\vec{\varphi}, \overrightarrow{g_{1}}, \overrightarrow{g_{2}}$ are linearly independent vectors (typically $\vec{\varphi}=\overrightarrow{g_{3}} \odot(1,1, g)$ where $\vec{\varphi}=$ \left.${\overrightarrow{g_{1}}}^{\xi_{1}} \odot{\overrightarrow{g_{2}}}^{\xi_{2}}\right)$ whereas, in the WI setting, choosing $\vec{\varphi}={\overrightarrow{g_{1}}}^{\xi_{1}} \odot{\overrightarrow{g_{2}}}^{\xi_{2}}$ gives a perfectly hiding commitment since $\vec{C}$ is always a BBS encryption of $1_{\mathbb{G}}$.

To prove that committed variables satisfy a set of relations, the GS techniques replace variables by the corresponding commitments in each relation. The whole proof consists of one commitment per variable and one proof element (made of a constant number of group elements) per relation.

Such proofs are available for pairing-product relations, which are of the type

$$
\prod_{i=1}^{n} e\left(\mathcal{A}_{i}, \mathcal{X}_{i}\right) \cdot \prod_{i=1}^{n} \cdot \prod_{j=1}^{n} e\left(\mathcal{X}_{i}, \mathcal{X}_{j}\right)^{a_{i j}}=t_{T}
$$


for variables $\mathcal{X}_{1}, \ldots, \mathcal{X}_{n} \in \mathbb{G}$ and constants $t_{T} \in \mathbb{G}_{T}, \mathcal{A}_{1}, \ldots, \mathcal{A}_{n} \in \mathbb{G}, a_{i j} \in \mathbb{G}$, for $i, j \in\{1, \ldots, n\}$. Efficient proofs also exist for multi-exponentiation equations

$$
\prod_{i=1}^{m} \mathcal{A}_{i}^{y_{i}} \cdot \prod_{j=1}^{n} \mathcal{X}_{j}^{b_{j}} \cdot \prod_{i=1}^{m} \cdot \prod_{j=1}^{n} \mathcal{X}_{j}^{y_{i} \gamma_{i j}}=T
$$

for variables $\mathcal{X}_{1}, \ldots, \mathcal{X}_{n} \in \mathbb{G}, y_{1}, \ldots, y_{m} \in \mathbb{Z}_{p}$ and constants $T, \mathcal{A}_{1}, \ldots, \mathcal{A}_{m} \in \mathbb{G}$, $b_{1}, \ldots, b_{n} \in \mathbb{Z}_{p}$ and $\gamma_{i j} \in \mathbb{G}$, for $i \in\{1, \ldots, m\}, j \in\{1, \ldots, n\}$.

Multi-exponentiation equations admit zero-knowledge proofs at no additional cost. On a simulated CRS (prepared for the WI setting), a trapdoor makes it is possible to simulate proofs without knowing witnesses and simulated proofs are perfectly indistinguishable from real proofs. As for pairing-product equations, zero-knowledge proofs are often possible but usually come at some expense. In the paper, we only resort to such NIZK simulators in one occasion.

In both cases, proofs for quadratic equations cost 9 group elements. Linear pairing-product equations (when $a_{i j}=0$ for all $i, j$ ) take 3 group elements each. Linear multi-exponentiation equations of the type $\prod_{j=1}^{n} \mathcal{X}_{j}^{b_{j}}=T$ (resp. $\prod_{i=1}^{m} \mathcal{A}_{i}^{y_{i}}=T$ ) demand 3 (resp. 2) group elements.

\section{$3 \quad$ Building Blocks}

Our certification scheme uses a trapdoor commitment to group elements as an important ingredient to dispense with proofs of knowledge of users' private keys.

\subsection{A Trapdoor Commitment to Group Elements}

We need a trapdoor commitment scheme that allows committing to elements of a group $\mathbb{G}$ where bilinear map arguments are taken. Commitments will have to be themselves elements of $\mathbb{G}$, which prevents us from using Groth's scheme [25] where commitments lie in the range $\mathbb{G}_{T}$ of the pairing.

Such commitments can be obtained using the perfectly hiding Groth-Sahai commitment based on the linear assumption recalled in section 2.3. This commitment uses a common reference string describing a prime order group $\mathbb{G}$ and a generator $f \in \mathbb{G}$. The commitment key consists of vectors $\left(\overrightarrow{f_{1}}, \overrightarrow{f_{2}}, \overrightarrow{f_{3}}\right)$ chosen as $\overrightarrow{f_{1}}=\left(f_{1}, 1, f\right), \overrightarrow{f_{2}}=\left(1, f_{2}, f\right)$ and $\overrightarrow{f_{3}}=\vec{f}_{1}^{\xi_{1}} \odot \vec{f}_{2}^{\xi_{2}} \odot(1,1, f)^{\xi_{3}}$, with $f_{1}, f_{2} \stackrel{\$}{\leftarrow}$, $\xi_{1}, \xi_{2}, \xi_{3} \stackrel{\$}{\leftarrow} \mathbb{Z}_{p}^{*}$. To commit to $X$, the sender picks $\phi_{1}, \phi_{2}, \phi_{3} \stackrel{\$}{\leftarrow} \mathbb{Z}_{p}^{*}$ and sets $\vec{C}_{X}=(1,1, X) \odot \vec{f}_{1}^{\phi_{1}} \odot{\overrightarrow{f_{2}}}^{\phi_{2}} \odot{\overrightarrow{f_{3}}}^{\phi_{3}}$, which, if $\vec{f}_{3}$ is parsed as $\left(f_{3,1}, f_{3,2}, f_{3,3}\right)$, can be written $\vec{C}_{X}=\left(f_{1}^{\phi_{1}} \cdot f_{3,1}^{\phi_{3}}, f_{2}^{\phi_{2}} \cdot f_{3,2}^{\phi_{3}}, X \cdot f^{\phi_{1}+\phi_{2}} \cdot f_{3,3}^{\phi_{3}}\right)$. Due to the use of GS proofs, commitment openings need to only consist of group elements (and no scalar). To open $\vec{C}_{X}=\left(C_{1}, C_{2}, C_{3}\right)$, the sender reveals $\left(D_{1}, D_{2}, D_{3}\right)=\left(f^{\phi_{1}}, f^{\phi_{2}}, f^{\phi_{3}}\right)$ and $X$. The receiver is convinced that the committed value was $X$ by checking that

$$
\left\{\begin{array}{l}
e\left(C_{1}, f\right)=e\left(f_{1}, D_{1}\right) \cdot e\left(f_{3,1}, D_{3}\right) \\
e\left(C_{2}, f\right)=e\left(f_{2}, D_{2}\right) \cdot e\left(f_{3,2}, D_{3}\right) \\
e\left(C_{3}, f\right)=e\left(X \cdot D_{1} \cdot D_{2}, f\right) \cdot e\left(f_{3,3}, D_{3}\right) .
\end{array}\right.
$$


If a cheating sender can come up with distinct openings of $\vec{C}_{X}$, we can easily solve a $S 2 P$ instance $\left(g_{1}, g_{2}, g_{1, c}, g_{2, d}\right)$. Namely, the commitment key is set as $\left(f_{1}, f_{2}, f_{3,1}, f_{3,2}\right)=\left(g_{1}, g_{2}, g_{1, c}, g_{2, d}\right)$ and $f, f_{3,3}$ are chosen at random. When the adversary outputs $\left(X,\left(D_{1}, D_{2}, D_{3}\right)\right)$ and $\left(X^{\prime},\left(D_{1}^{\prime}, D_{2}^{\prime}, D_{3}^{\prime}\right)\right)$, we must simultaneously have $e\left(f_{1}, D_{1} / D_{1}^{\prime}\right)=e\left(f_{3,1}, D_{3}^{\prime} / D_{3}\right), e\left(f_{2}, D_{2} / D_{2}^{\prime}\right)=e\left(f_{3,2}, D_{3}^{\prime} / D_{3}\right)$ and $e\left(\left(X D_{1} D_{2}\right) /\left(X^{\prime} D_{1}^{\prime} D_{2}^{\prime}\right), f\right)=e\left(f_{3,3}, D_{3}^{\prime} / D_{3}\right)$. Hence, setting $u=D_{1} / D_{1}^{\prime}$, $v=D_{2} / D_{2}^{\prime}$ and $w=D_{3}^{\prime} / D_{3}$ solves the S2P problem as $(u, v, w)$ can only be trivial if $X^{\prime}=X$.

Using the trapdoor $\left(\xi_{1}, \xi_{2}, \xi_{3}\right)$, the receiver can equivocate commitments. Given a commitment $\vec{C}_{X}$ and its opening $\left(X,\left(D_{1}, D_{2}, D_{3}\right)\right)$, one can trapdoor open $\vec{C}_{X}$ to any other $X^{\prime} \in \mathbb{G}$ (and without knowing $\log _{g}\left(X^{\prime}\right)$ ) by computing

$$
D_{1}^{\prime}=D_{1} \cdot\left(X^{\prime} / X\right)^{\xi_{1} / \xi_{3}}, \quad D_{2}^{\prime}=D_{2} \cdot\left(X^{\prime} / X\right)^{\xi_{2} / \xi_{3}}, \quad D_{3}^{\prime}=\left(X / X^{\prime}\right)^{1 / \xi_{3}} \cdot D_{3} .
$$

\subsection{A Public Key Certification Scheme}

We use a primitive that we call non-interactive certification scheme, which can be viewed as a signature scheme that only allows signing public keys from a specific public key space $\mathcal{P} \mathcal{K}$. These keys should be signed while retaining algebraic properties that make it possible to prove knowledge of a public key and its corresponding certificate in an efficient way. In particular, signing hashed public keys is proscribed. In the interactive setting, several papers (e.g., [5|24]) describe efficient interactive protocols where a public key is jointly generated by a user and a certification authority in such a way that the user eventually obtains a certified public key and no one else learns the underlying private key. In this paper, we aim at minimizing the amount of interaction and let users generate their public key entirely on their own before requesting their certification. Ideally, we would like to be able to sign public keys without even requiring users to prove knowledge of their private key and, in particular, without having to first rewind a proof of knowledge so as to extract the user's private key in the security proof.

A certification scheme consists of algorithms (Setup, Certify, CertVerify). The first one is run by a certification authority (CA) that, on input of global parameters cp, generates a key pair $(S K, P K) \leftarrow$ Setup $(\mathrm{cp})$. On input of cp, $S K$ and a user's public key pk, Certify generates a certificate cert $_{\mathrm{pk}}$. The procedure Verify takes as input $\mathrm{cp}, P K$, pk and cert $\mathrm{pk}_{\mathrm{pk}}$ and outputs either 0 or 1 .

Correctness mandates that CertVerify $\left(\mathrm{cp}, P K, \mathrm{pk}\right.$, cert $\left._{\mathrm{pk}}\right)=1$ when $^{\text {cert }} \mathrm{pk} \leftarrow$ Certify (cp, $S K$, pk). The (strong) unforgeability [1] requirement is the same as in signature schemes. The adversary is supplied with a CA's public key $P K$ and access to a certification oracle $\operatorname{Certify}(S K,$.$) that can be queried for arbitrary$ public keys pk $\in \mathcal{P} \mathcal{K}$. Her goal is to produce a new pair $\left(\mathrm{pk}^{*}\right.$, cert $\left._{\mathrm{pk}^{*}}^{*}\right)\left(\right.$ i.e., if $\mathrm{pk}^{*}$ was queried to Certify $(S K,$.$\left.) , the output must have been different from cert \mathrm{pk}^{*}\right)$.

In the description hereafter, we assume common public parameters cp consisting of of bilinear groups $\left(\mathbb{G}, \mathbb{G}_{T}\right)$ of prime order $p>2^{\lambda}$, for a security parameter $\lambda$, and a generator $g \stackrel{\&}{\leftarrow}$. We also assume that certified public keys always consist of a fixed number $n$ of group elements $\left(\right.$ i.e., $\left.\mathcal{P} \mathcal{K}=\mathbb{G}^{n}\right)$. 
Intuition. The scheme borrows from the Boyen-Waters group signature [10] in the use of the HSDH assumption. A simplified version involves a CA that holds a public key $P K=\left(\Omega=g^{\omega}, A=(g, g)^{\alpha}, u, u_{0}, u_{1}=g^{\beta_{1}}, \ldots, u_{n}=g^{\beta_{n}}\right)$, for private elements $S K=\left(\omega, \alpha, \beta_{1}, \ldots, \beta_{n}\right)$, where $n$ denotes the number of groups elements that certified public keys consist of. To certify a public key pk $=\left(X_{1}=g^{x_{1}}, \ldots, X_{n}=g^{x_{n}}\right)$, the CA chooses an exponent $c_{\mathrm{ID}} \stackrel{\$}{\leftarrow} \mathbb{Z}_{p}^{*}$ and computes $S_{1}=\left(g^{\alpha}\right)^{1 /\left(\omega+c_{\mathrm{ID}}\right)}, S_{2}=g^{c_{\mathrm{ID}}}, S_{3}=u^{c_{\mathrm{ID}}}, S_{4}=\left(u_{0} \cdot \prod_{i=1}^{n} X_{i}^{\beta_{i}}\right)^{c_{\mathrm{ID}}}$ and $S_{5}=\left(S_{5,1}, \ldots, S_{5, n}\right)=\left(X_{1}^{c_{\mathrm{ID}}}, \ldots, X_{n}^{c_{\mathrm{ID}}}\right)$. Verification then checks whether $e\left(S_{1}, \Omega \cdot S_{2}\right)=A$ and $e\left(S_{2}, u\right)=e\left(g, S_{3}\right)$ as in [10]. It must also be checked that $e\left(S_{4}, g\right)=e\left(u_{0}, S_{2}\right) \cdot \prod_{i=1}^{n} e\left(u_{i}, S_{5, i}\right)$ and $e\left(S_{5, i}, g\right)=e\left(X_{i}, S_{2}\right)$ for $i=1, \ldots, n$.

The security of this simplified scheme can only be proven if, when answering certification queries, the simulator can control the private keys $\left(x_{1}, \ldots, x_{n}\right)$ and force them to be random values of its choice. To allow the simulator to sign arbitrary public keys without knowing the private keys, we modify the scheme so that the $\mathrm{CA}$ rather signs commitments (calculated as in the trapdoor commitment of section 3.1) to public key elements $X_{1}, \ldots, X_{n}$. In the security proof, the simulator first generates a signature on $n$ commitments $\vec{C}_{i}=\left(C_{i, 1}, C_{i, 2}, C_{i, 3}\right)$ to $1_{\mathbb{G}}$ that are all generated in such a way that it $\operatorname{knows} \log _{g}\left(C_{i, j}\right)$ for $i=1, \ldots, n$ and $j=1,2,3$. Using the trapdoor of the commitment scheme, it can then open $\vec{C}_{i}$ to any arbitrary public key element $X_{i}$ without $\operatorname{knowing} \log _{g}\left(X_{i}\right)$.

This use of the trapdoor commitment is reminiscent of a technique (notably used in [18) to construct signature schemes in the standard model using chameleon hash functions 32: the simulator first signs messages of its choice using a basic signature scheme and then "equivocates" the chameleon hashes to make them correspond to adversarially-chosen messages.

Setup $(\mathrm{cp})$ : given common public parameters cp $=\left\{g, \mathbb{G}, \mathbb{G}_{T}\right\}$, select $u, u_{0} \stackrel{\$}{\leftarrow}$ $\mathbb{G}, \alpha, \omega \stackrel{\$}{\leftarrow} \mathbb{Z}_{p}^{*}$ and set $A=e(g, g)^{\alpha}, \Omega=g^{\omega}$. Pick $\beta_{i, 1}, \beta_{i, 2}, \beta_{i, 3} \stackrel{\$}{\leftarrow} \mathbb{Z}_{p}^{*}$ and set $\bar{u}_{i}=\left(u_{i, 1}, u_{i, 2}, u_{i, 3}\right)=\left(g^{\beta_{i, 1}}, g^{\beta_{i, 2}}, g^{\beta_{i, 3}}\right)$ for $i=1, \ldots, n$. Choose $f, f_{1}, f_{2}, f_{3,1}, f_{3,2}, f_{3,3} \stackrel{\$}{\leftarrow} \mathbb{G}$ that define a commitment key consisting of vectors $\overrightarrow{f_{1}}=\left(f_{1}, 1, f\right), \overrightarrow{f_{2}}=\left(1, f_{2}, f\right)$ and $\overrightarrow{f_{3}}=\left(f_{3,1}, f_{3,2}, f_{3,3}\right)$. Define the private/public key pair as $S K=\left(\alpha, \omega,\left\{\bar{\beta}_{i}=\left(\beta_{i, 1}, \beta_{i, 2}, \beta_{i, 3}\right)\right\}_{i=1, \ldots, n}\right)$ and

$$
P K=\left(\mathbf{f}=\left(\overrightarrow{f_{1}}, \overrightarrow{f_{2}}, \overrightarrow{f_{3}}\right), A=e(g, g)^{\alpha}, \Omega=g^{\omega}, u, u_{0},\left\{\bar{u}_{i}\right\}_{i=1, \ldots, n}\right) .
$$

$\operatorname{Certify}(\mathrm{cp}, S K, \mathrm{pk})$ : parse $S K$ as $\left(\alpha, \omega,\left\{\bar{\beta}_{i}\right\}_{i=1, \ldots, n}\right)$, pk as $\left(X_{1}, \ldots, X_{n}\right)$ and do the following.

1. For each $i \in\{1, \ldots, n\}$, pick $\phi_{i, 1}, \phi_{i, 2}, \phi_{i, 3} \stackrel{\$}{\leftarrow} \mathbb{Z}_{p}^{*}$ and compute a commitment $C_{i}=\left(C_{i, 1}, C_{i, 2}, C_{i, 3}\right)=\left(f_{1}^{\phi_{i, 1}} \cdot f_{3,1}^{\phi_{i, 3}}, f_{2}^{\phi_{i, 2}} \cdot f_{3,2}^{\phi_{i, 3}}, X_{i} \cdot f^{\phi_{i, 1}+\phi_{i, 2}} \cdot f_{3,3}^{\phi_{i, 3}}\right)$ and the matching de-commitment $\left(D_{i, 1}, D_{i, 2}, D_{i, 3}\right)=\left(f^{\phi_{i, 1}}, f^{\phi_{i, 2}}, f^{\phi_{i, 3}}\right)$.

2. Choose $c_{\mathrm{ID}} \stackrel{\$}{\leftarrow} \mathbb{Z}_{p}^{*}$, compute $S_{1}=\left(g^{\alpha}\right)^{1 /\left(\omega+c_{\mathrm{ID}}\right)}, S_{2}=g^{c_{\mathrm{ID}}}, S_{3}=u^{c_{\mathrm{ID}}}$ and

$$
\begin{aligned}
& S_{4}=\left(u_{0} \cdot \prod_{i=1}^{n}\left(C_{i, 1}^{\beta_{i, 1}} \cdot C_{i, 2}^{\beta_{i, 2}} \cdot C_{i, 3}^{\beta_{i, 3}}\right)\right)^{c_{\mathrm{ID}}} \\
& S_{5}=\left\{\left(S_{5, i, 1}, S_{5, i, 2}, S_{5, i, 3}\right)\right\}_{i=1, \ldots, n}=\left\{\left(C_{i, 1}^{c_{\mathrm{ID}}}, C_{i, 2}^{c_{\mathrm{ID}}}, C_{i, 3}^{c_{\mathrm{ID}}}\right)\right\}_{i=1, \ldots, n}
\end{aligned}
$$


Return cert pk $=\left(\left\{\left(C_{i, 1}, C_{i, 2}, C_{i, 3}\right),\left(D_{i, 1}, D_{i, 2}, D_{i, 3}\right)\right\}_{i=1, \ldots, n}, S_{1}, S_{2}, S_{3}, S_{4}, S_{5}\right)$.

CertVerify $\left(\mathrm{cp}, P K\right.$, pk, cert $\left._{\mathrm{pk}}\right)$ : parse pk as $\left(X_{1}, \ldots, X_{n}\right)$ and cert $_{\mathrm{pk}}$ as above. Return 1 if, for $i=1, \ldots, n$, it holds that $X_{i} \in \mathbb{G}$ and

$$
\begin{aligned}
& e\left(C_{i, 1}, f\right)=e\left(f_{1}, D_{i, 1}\right) \cdot e\left(f_{3,1}, D_{i, 3}\right) \\
& e\left(C_{i, 2}, f\right)=e\left(f_{2}, D_{i, 2}\right) \cdot e\left(f_{3,2}, D_{i, 3}\right) \\
& e\left(C_{i, 3}, f\right)=e\left(X_{i} \cdot D_{i, 1} \cdot D_{i, 2}, f\right) \cdot e\left(f_{3,3}, D_{i, 3}\right),
\end{aligned}
$$

and if the following checks are also satisfied. Otherwise, return 0.

$$
\begin{aligned}
& e\left(S_{1}, \Omega \cdot S_{2}\right)=A \\
& e\left(S_{2}, u\right)=e\left(g, S_{3}\right) \\
& e\left(S_{4}, g\right)=e\left(u_{0}, S_{2}\right) \cdot \prod_{i=1}^{n}\left(e\left(u_{i, 1}, S_{5, i, 1}\right) \cdot e\left(u_{i, 2}, S_{5, i, 2}\right) \cdot e\left(u_{i, 3}, S_{5, i, 3}\right)\right), \\
& e\left(S_{5, i, j}, g\right)=e\left(C_{i, j}, S_{2}\right) \quad \text { for } i=1, \ldots, n, j=1,2,3
\end{aligned}
$$

A certificate comprises $9 n+4$ group elements. It would be interesting to avoid this linear dependency on $n$ without destroying the algebraic properties that render the scheme compatible with Groth-Sahai proofs.

Regarding the security of this scheme, the idea of the proof of the following theorem is sketched in appendix $\mathrm{A}$. Due to space limitation, the complete proof is detailed in the full version of the paper.

Theorem 1. The scheme is a secure non-interactive certification system if the $H S D H$, FlexDH and S2P problems are all hard in $\mathbb{G}$.

We believe that the above certification scheme is of interest in its own right. For instance, it can be used to construct non-frameable group signatures that are secure in the concurrent join model of 30 without resorting to random oracles. To the best of our knowledge, the Kiayias-Yung construction [30] has remained the only scalable group signature where joining supports concurrency at both ends while requiring the smallest amount of interaction. In the standard model, our certification scheme thus appears to provide the first 2 way to achieve the same result. In this case, we have $n=1$ (since prospective group members only need to certify one group element if non-frameability is ensured by signing messages as in Groth's group signature 24]) so that membership certificates comprise 13 group elements and their shape is fully compatible with GS proofs.

\footnotetext{
${ }^{2}$ Non-frameable group signatures described in 199 achieve concurrent security by having the prospective user generate an extractable commitment to some secret exponent (which the simulator can extract without rewinding using the trapdoor of the commitment) and prove that the committed value is the discrete log. of a public value. In the standard model, this technique requires interaction and the proof should be simulatable in zero-knowledge when proving security against framing attacks. Another technique 21 requires users to prove knowledge of their secret exponent using Groth-Sahai non-interactive proofs. It is nevertheless space-demanding as each bit of committed exponent requires its own extractable GS commitment.
} 


\subsection{Public Key Encryption Schemes Based on the Linear Problem}

We need cryptosystems based on the DLIN assumption. The first one is Shacham's variant [37] of Cramer-Shoup [17] and, since it is key-private [3], we use it to encrypt witnesses. We also use Kiltz's tag-based encryption (TBE) scheme [31, where the validity of ciphertexts is publicly verifiable, to encrypt receivers' public keys under the public key of the opening authority.

Shacham's Linear CRAmer-Shoup. If we assume public generators $g_{1}, g_{2}, g$ that are parts of public parameters, each receiver's public key is made of $n=6$ group elements

$$
\begin{array}{lll}
X_{1}=g_{1}^{x_{1}} g^{x} & X_{3}=g_{1}^{x_{3}} g^{y} & X_{5}=g_{1}^{x_{5}} g^{z} \\
X_{2}=g_{2}^{x_{2}} g^{x} & X_{4}=g_{2}^{x_{4}} g^{y} & X_{6}=g_{2}^{x_{6}} g^{z} .
\end{array}
$$

To encrypt $m \in \mathbb{G}$ under the label $L$, the sender picks $r, s \stackrel{\$}{\leftarrow} \mathbb{Z}_{p}^{*}$ and computes $\psi_{\mathrm{CS}}=\left(U_{1}, U_{2}, U_{3}, U_{4}, U_{5}\right)=\left(g_{1}^{r}, g_{2}^{s}, g^{r+s}, m \cdot X_{5}^{r} X_{6}^{s},\left(X_{1} X_{3}^{\alpha}\right)^{r} \cdot\left(X_{2} X_{4}^{\alpha}\right)^{s}\right)$,

where $\alpha=H\left(U_{1}, U_{2}, U_{3}, U_{4}, L\right) \in \mathbb{Z}_{p}^{*}$ is a collision-resistant hash 3 . Given $\left(\psi_{\mathrm{CS}}, L\right)$, the receiver computes $\alpha$. He returns $\perp$ if $U_{5} \neq U_{1}^{x_{1}+\alpha x_{3}} U_{2}^{x_{2}+\alpha x_{4}} U_{3}^{x+\alpha y}$ and $m=U_{4} /\left(U_{1}^{x_{5}} U_{2}^{x_{6}} U_{3}^{z}\right)$ otherwise.

Kiltz's Tag-Based Encryption Scheme. In [31, Kiltz described a TBE scheme based on the same assumption. The public key is $\left(Y_{1}, Y_{2}, Y_{3}, Y_{4}\right)=$ $\left(g^{y_{1}}, g^{y_{2}}, g^{y_{3}}, g^{y_{4}}\right)$ if $g \in \mathbb{G}$ is part of public parameters. To encrypt $m \in \mathbb{G}$ under a tag $t \in \mathbb{Z}_{p}^{*}$, the sender picks $w_{1}, w_{2} \stackrel{\$}{\leftarrow} \mathbb{Z}_{p}^{*}$ and computes

$$
\psi_{\mathrm{K}}=\left(V_{1}, V_{2}, V_{3}, V_{4}, V_{5}\right)=\left(Y_{1}^{w_{1}}, Y_{2}^{w_{2}},\left(g^{t} Y_{3}\right)^{w_{1}},\left(g^{t} Y_{4}\right)^{w_{2}}, m \cdot g^{w_{1}+w_{2}}\right)
$$

To decrypt $\psi_{\mathrm{K}}$, the receiver checks that $V_{3}=V_{1}^{\left(t+y_{3}\right) / y_{1}}, V_{4}=V_{2}^{\left(t+y_{4}\right) / y_{2}}$. If so, it outputs the plaintext $m=V_{5} /\left(V_{1}^{1 / y_{1}} V_{2}^{1 / y_{2}}\right)$. Unlike $\psi_{\mathrm{CS}}$, the well-formedness of $\psi_{\mathrm{K}}$ is publicly verifiable in bilinear groups. The Canetti-Halevi-Katz [15] paradigm turns this scheme into a full-fledged CCA2 scheme by deriving the tag $t$ from the verification key VK of a one-time signature, the private key SK of which is used to $\operatorname{sign}\left(V_{1}, V_{2}, V_{3}, V_{4}, V_{5}\right)$.

\section{A GE Scheme with Non-interactive Proofs}

We build a non-interactive group encryption scheme for the Diffie-Hellman relation $\mathcal{R}=\{(X, Y), W\}$ where $e(g, W)=e(X, Y)$, for which the keys are $\mathrm{pk}_{\mathcal{R}}=\left\{\mathbb{G}, \mathbb{G}_{T}, g\right\}$ and $\mathrm{sk}_{\mathcal{R}}=\varepsilon$.

The construction slightly departs from the modular design of 29 in that commitments to the receiver's public key and certificate are part of the proof (instead of the ciphertext), which simplifies the proof of message-security. The security of the scheme eventually relies on the HSDH, FlexDH and DLIN assumptions. All security proofs are available in the full version of the paper.

${ }^{3}$ The proof of CCA2-security [17/37] only requires a universal one-way hash function (UOWHF) 34 but collision-resistance is required by the proof of key-privacy in 3 . 
$\operatorname{SETUP}_{\text {init }}(\lambda)$ : choose bilinear groups $\left(\mathbb{G}, \mathbb{G}_{T}\right)$ of order $p>2^{\lambda}, g \stackrel{\$}{\leftarrow} \mathbb{G}$ and $g_{1}=g^{\alpha_{1}}, g_{2}=g^{\alpha_{2}}$ with $\alpha_{1}, \alpha_{2} \stackrel{\$}{\leftarrow} \mathbb{Z}_{p}^{*}$. Define $\overrightarrow{g_{1}}=\left(g_{1}, 1, g\right), \overrightarrow{g_{2}}=\left(1, g_{2}, g\right)$ and $\overrightarrow{g_{3}}=\overrightarrow{g_{1}} \xi_{1} \odot \overrightarrow{g_{2}} \xi_{2}$ with $\xi_{1}, \xi_{2} \stackrel{\$}{\leftarrow} \mathbb{Z}_{p}^{*}$, which form a CRS $\mathbf{g}=\left(\overrightarrow{g_{1}}, \overrightarrow{g_{2}}, \overrightarrow{g_{3}}\right)$ for the perfect soundness setting. Select a strongly unforgeable (as defined in [1]) one time signature scheme $\Sigma=(\mathcal{G}, \mathcal{S}, \mathcal{V})$ and a random member $H:\{0,1\}^{*} \rightarrow \mathbb{Z}_{p}$ of a collision-resistant hash family. Public parameters consists of param $=\left\{\lambda, \mathbb{G}, \mathbb{G}_{T}, g, \mathbf{g}, \Sigma, H\right\}$.

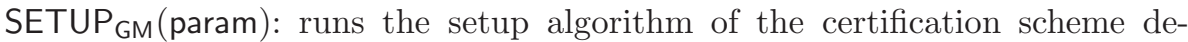
scribed in section 3.2 with $n=6$. The obtained public key consists of $\mathrm{pk}_{\mathrm{GM}}=\left(\mathbf{f}, A=e(g, g)^{\alpha}, \Omega=g^{\omega}, u, u_{0},\left\{\bar{u}_{i}\right\}_{i=1, \ldots, 6}\right)$ and the matching private key is sk $\mathrm{GM}=\left(\alpha, \omega,\left\{\bar{\beta}_{i}=\left(\beta_{i, 1}, \beta_{i, 2}, \beta_{i, 3}\right)\right\}_{i=1, \ldots, 6}\right)$.

$\operatorname{SETUP}_{\mathrm{OA}}($ param $)$ : generates pk $\mathrm{OA}=\left(Y_{1}, Y_{2}, Y_{3}, Y_{4}\right)=\left(g^{y_{1}}, g^{y_{2}}, g^{y_{3}}, g^{y_{4}}\right)$, as a public key for Kiltz's tag-based encryption scheme [31], and the corresponding private key as skoA $=\left(y_{1}, y_{2}, y_{3}, y_{4}\right)$.

JOIN: the user sends a linear Cramer-Shoup public key pk $=\left(X_{1}, \ldots, X_{6}\right) \in \mathbb{G}^{6}$ to the GM and obtains a certificate

$$
\operatorname{cert}_{\mathrm{pk}}=\left(\left\{\left(C_{i, 1}, C_{i, 2}, C_{i, 3}\right),\left(D_{i, 1}, D_{i, 2}, D_{i, 3}\right)\right\}_{i=1, \ldots, 6}, S_{1}, S_{2}, S_{3}, S_{4}, S_{5}\right) .
$$

ENC $\left(\mathrm{pk}_{\mathrm{GM}}, \mathrm{pk}_{\mathrm{OA}}, \mathrm{pk}\right.$, cert $\left._{\mathrm{pk}}, W, L\right)$ : to encrypt $W \in \mathbb{G}$ such that $((X, Y), W) \in \mathcal{R}$ (for public elements $X, Y \in \mathbb{G}$ ), parse $\mathrm{pk}_{\mathrm{GM}}$, $\mathrm{pk}_{\mathrm{OA}}$ and $\mathrm{pk}$ as above and do the following.

1. Generate a one-time signature key pair $(\mathrm{SK}, \mathrm{VK}) \leftarrow \mathcal{G}(\lambda)$.

2. Choose $r, s \stackrel{\$}{\leftarrow} \mathbb{Z}_{p}^{*}$ and compute a linear CS encryption of $W$, the result of which is denoted by $\psi_{\mathrm{CS}}$, under the label $L_{1}=L \| \mathrm{VK}$ as per section 3.3 (and using the collision-resistant hash function specified by param).

3. For $i=1, \ldots, 6$, choose $w_{i, 1}, w_{i, 2} \stackrel{\$}{\leftarrow} \mathbb{Z}_{p}^{*}$ and encrypt $X_{i}$ under pk $\mathrm{OA}_{\mathrm{OA}}$ using Kiltz's TBE with the tag VK as described in section 3.3 . Let $\psi_{\mathrm{K}_{i}}$ be the ciphertexts.

4. Set the ciphertext $\psi$ as $\psi=\mathrm{VK}\left\|\psi_{\mathrm{CS}}\right\| \psi_{\mathrm{K}_{1}}\|\cdots\| \psi_{\mathrm{K}_{6}} \| \sigma$ where $\sigma$ is obtained as $\sigma=\mathcal{S}\left(\mathrm{SK},\left(\psi_{\mathrm{CS}}\left\|\psi_{\mathrm{K}_{1}}\right\| \cdots\left\|\psi_{\mathrm{K}_{6}}\right\| L\right)\right)$.

Return $(\psi, L)$ and coins $_{\psi}$ consist of $\left\{\left(w_{i, 1}, w_{i, 2}\right)\right\}_{i=1, \ldots, 6},(r, s)$. If the onetime signature of 23 is used, VK and $\sigma$ take 3 and 2 group elements, respectively, so that $\psi$ comprises 40 group elements.

$\mathcal{P}\left(\mathrm{pk}_{\mathrm{GM}}, \mathrm{pk}_{\mathrm{OA}}, \mathrm{pk}, \operatorname{cert}_{\mathrm{pk}},(X, Y), W, \psi, L, \operatorname{coins}_{\psi}\right):$ parse $\mathrm{pk}_{\mathrm{GM}}, \mathrm{pk}_{\mathrm{OA}}$, pk and $\psi$ as above. Conduct the following steps.

1. Generate commitments (as explained in section 2.3) to the $9 n+4=58$

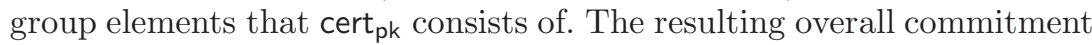
com $_{\text {cert }_{\mathrm{pk}}}$ contains 184 group elements.

2. Generate commitments to the public key elements $\mathrm{pk}=\left(X_{1}, \ldots, X_{6}\right)$ and obtain $\operatorname{com}_{\mathrm{pk}}=\left\{\operatorname{com}_{X_{i}}\right\}_{i=1, \ldots, 6}$, which consists of 18 group elements.

3. Generate a proof $\pi_{\mathrm{cert}}$ that $\operatorname{com}_{\mathrm{cert}}$ is a commitment to a valid certificate for the public key contained in $\operatorname{com}_{\mathrm{pk}}$. For each $i=1, \ldots, 6$, 
relations (1)-(3) cost 9 elements to prove (and thus 54 elements altogether). The quadratic equation (4) takes 9 elements and linear ones (5)-(6) both require 3 elements. Finally, (7) is a set of 18 linear equations which demand 54 elements altogether. The whole proof $\pi_{\text {cert }}$ thus takes 123 group elements.

4. For $i=1, \ldots, 6$, generate a NIZK proof $\pi_{e q-k e y, i}$ that $\operatorname{com}_{X_{i}}$ (which is part of $\left.c o m_{\mathrm{pk}}\right)$ and $\psi_{\mathrm{K}_{i}}$ are encryptions of the same $X_{i}$. If $\psi_{\mathrm{K}_{i}}$ comprises $\left(V_{i, 1}, V_{i, 2}, V_{i, 5}\right)=\left(Y_{1}^{w_{i, 1}}, Y_{2}^{w_{i, 2}}, X_{i} \cdot g^{w_{i, 1}+w_{i, 2}}\right)$ and $c o m_{X_{i}}$ is parsed as $\left(c_{X_{i 1}}, c_{X_{i 2}}, c_{X_{i 3}}\right)=\left(g_{1}^{\theta_{i 1}} \cdot g_{3,1}^{\theta_{i 3}}, g_{2}^{\theta_{i 2}} \cdot g_{3,2}^{\theta_{i 3}}, X_{i} \cdot g^{\theta_{i 1}+\theta_{i 2}} \cdot g_{3,3}^{\theta_{i 3}}\right)$, where $w_{i, 1}, w_{i, 2} \in$ coins $_{\psi}, \theta_{i 1}, \theta_{i 2}, \theta_{i 3} \in \mathbb{Z}_{p}^{*}$ and $\overrightarrow{g_{3}}=\left(g_{3,1}, g_{3,2}, g_{3,3}\right)$, this amounts to prove knowledge of values $w_{i, 1}, w_{i, 2}, \theta_{i 1}, \theta_{i 2}, \theta_{i 3}$ such that

$$
\begin{aligned}
\left(\frac{V_{i, 1}}{c_{X_{i 1}}}, \frac{V_{i, 2}}{c_{X_{i 2}}}, \frac{V_{i, 3}}{c_{X_{i 3}}}\right)= & \left(Y_{1}^{w_{i, 1}} \cdot g_{1}^{-\theta_{i 1}} \cdot g_{3,1}^{-\theta_{i 3}},\right. \\
& \left.Y_{2}^{w_{i, 2}} \cdot g_{2}^{-\theta_{i 2}} \cdot g_{3,2}^{-\theta_{i 3}}, g^{w_{i, 1}+w_{i, 2}-\theta_{i 1}-\theta_{i 2}} \cdot g_{3,3}^{-\theta_{i 3}}\right) .
\end{aligned}
$$

Committing to $w_{i, 1}, w_{i, 2}, \theta_{i 1}, \theta_{i 2}, \theta_{i 3}$ introduces 90 group elements whereas the above relations only require two elements each. Overall, proof elements $\pi_{e q-k e y, 1}, \ldots, \pi_{e q-k e y, 6}$ incur 126 elements.

5. Generate a NIZK proof $\pi_{\text {val-enc }}$ that $\psi_{\mathrm{CS}}=\left(U_{1}, U_{2}, U_{3}, U_{4}, U_{5}\right)$ is a valid $\mathrm{CS}$ encryption. This requires to commit to underlying encryption exponents $r, s \in$ coins $_{\psi}$ and prove that $U_{1}=g_{1}^{r}, U_{2}=g_{2}^{s}, U_{3}=g^{r+s}$ (which only takes 3 times 2 elements as base elements are public) and $U_{5}=\left(X_{1} X_{3}^{\alpha}\right)^{r}\left(X_{2} X_{4}^{\alpha}\right)^{s}$ (which takes 9 elements since base elements are themselves variables). Including commitments com $_{r}$ and com $_{s}$ to exponents $r$ and $s, \pi_{v a l-e n c}$ demands 21 group elements overall.

6. Generate a NIZK proof $\pi_{\mathcal{R}}$ that $\psi_{\text {CS }}$ encrypts a group element $W \in \mathbb{G}$ such that $((X, Y), W) \in \mathcal{R}$. To this end, generate a commitment $\operatorname{com}_{W}=$ $\left(c_{W, 1}, c_{W, 2}, c_{W, 3}\right)=\left(g_{1}^{\theta_{1}} \cdot g_{3,1}^{\theta_{3}}, g_{2}^{\theta_{2}} \cdot g_{3,2}^{\theta_{3}}, W \cdot g^{\theta_{1}+\theta_{2}} g_{3,3}^{\theta_{3}}\right)$ and prove that the underlying $W$ is the same as the one for which $U_{4}=W \cdot X_{5}^{r} X_{6}^{s}$ in $\psi_{\mathrm{CS}}$. In other words, prove knowledge of $r, s, \theta_{1}, \theta_{2}, \theta_{3}$ such that

$$
\begin{aligned}
\left(\frac{U_{1}}{c_{W, 1}}, \frac{U_{2}}{c_{W, 2}}, \frac{U_{4}}{c_{W, 3}}\right)=\left(g_{1}^{r-\theta_{1}} \cdot g_{3,1}^{-\theta_{3}},\right. & \\
& \left.g_{2}^{s-\theta_{2}} \cdot g_{3,2}^{-\theta_{3}}, g^{-\theta_{1}-\theta_{2}} \cdot g_{3,3}^{-\theta_{3}} \cdot X_{5}^{r} \cdot X_{6}^{s}\right) .
\end{aligned}
$$

Commitments to $r, s$ are already part of $\pi_{\text {val-enc. }}$. Committing to $\theta_{1}, \theta_{2}, \theta_{3}$ takes 9 elements. Proving the first two relations of (8) requires 4 elements whereas the third one is quadratic and its proof is 9 elements. Proving the linear pairing-product relation $e(g, W)=e(X, Y)$ in NIZK 4 demands 9 elements. Since $\pi_{\mathcal{R}}$ includes $\operatorname{com}_{W}$, it entails a total of 34 elements.

${ }^{4}$ It requires to introduce an auxiliary variable $\mathcal{X}$ and prove that $e(g, \mathcal{W})=e(\mathcal{X}, Y)$ and $\mathcal{X}=X$, for variables $\mathcal{W}, \mathcal{X}$ and constants $g, X, Y$. The two proofs take 3 elements each and 3 elements are needed to commit to $\mathcal{X}$. 
The proof $\pi_{\psi}=\operatorname{com}_{\text {cert }_{\mathrm{pk}}}\left\|\operatorname{com}_{\mathrm{pk}}\right\| \pi_{\text {cert }_{\mathrm{pk}}}\left\|\pi_{\text {eq-key }, 1}\right\| \cdots\left\|\pi_{\text {eq-key }, 6}\right\| \pi_{\text {val-enc }} \| \pi_{\mathcal{R}}$ eventually takes 516 elements.

$\mathcal{V}\left(\right.$ param, $\left.\psi, L, \pi_{\psi}, \mathrm{pk}_{\mathrm{GM}}, \mathrm{pk}_{\mathrm{OA}}\right)$ : parse $\mathrm{pk}_{\mathrm{GM}}, \mathrm{pk}_{\mathrm{OA}}, \mathrm{pk}, \psi$ and $\pi_{\psi}$ as above. Return 1 if and only if $\mathcal{V}\left(\mathrm{VK}, \sigma,\left(\psi_{\mathrm{CS}}\left\|\psi_{\mathrm{K}_{1}}\right\| \cdots\left\|\psi_{\mathrm{K}_{6}}\right\| L\right)\right)=1$, all proofs verify and if $\psi_{\mathrm{K}_{1}}, \ldots, \psi_{\mathrm{K}_{6}}$ are all valid tag-based encryptions w.r.t. the tag VK.

$\operatorname{DEC}($ sk, $\psi, L)$ : parse the ciphertext $\psi$ as $\mathrm{VK}\left\|\psi_{\mathrm{CS}}\right\| \psi_{\mathrm{K}_{1}}\|\cdots\| \psi_{\mathrm{K}_{6}} \| \sigma$. Return $\perp$ if $\mathcal{V}\left(\mathrm{VK}, \sigma,\left(\psi_{\mathrm{CS}}\left\|\psi_{\mathrm{K}_{1}}\right\| \cdots\left\|\psi_{\mathrm{K}_{6}}\right\| L\right)\right)=0$. Otherwise, use sk to decrypt $\left(\psi_{\mathrm{CS}}, L\right)$.

$\operatorname{OPEN}\left(\operatorname{sk}_{\mathrm{OA}}, \psi, L\right)$ : parse the ciphertext $\psi$ as $\mathrm{VK}\left\|\psi_{\mathrm{CS}}\right\| \psi_{\mathrm{K}_{1}}\|\cdots\| \psi_{\mathrm{K}_{6}} \| \sigma$. Return $\perp$ if $\psi_{\mathrm{K}_{1}}, \ldots, \psi_{\mathrm{K}_{6}}$ are not all valid TBE ciphertexts w.r.t. the tag VK or if $\mathcal{V}\left(\mathrm{VK}, \sigma,\left(\psi_{\mathrm{CS}}\left\|\psi_{\mathrm{K}_{1}}\right\| \cdots\left\|\psi_{\mathrm{K}_{6}}\right\| L\right)\right)=0$. Otherwise, decrypt $\psi_{\mathrm{K}_{1}}, \ldots, \psi_{\mathrm{K}_{6}}$ using skoA and return the resulting $\mathrm{pk}=\left(X_{1}, \ldots, X_{6}\right)$.

From an efficiency standpoint, the length of ciphertexts is about $1.25 \mathrm{kB}$ in an implementation using symmetric pairings with a 256-bit group order, which is more compact than in the Paillier-based scheme of 29 where ciphertexts take 2.5 $\mathrm{kB}$ using 1024-bit moduli. Moreover, our proofs only require $16.125 \mathrm{kB}$, which is significantly cheaper than in the original GE scheme [29], where interactive proofs reach a communication cost of $70 \mathrm{kB}$ to achieve a $2^{-50}$ knowledge error.

\section{References}

1. An, J.H., Dodis, Y., Rabin, T.: On the security of joint signature and encryption. In: Knudsen, L.R. (ed.) EUROCRYPT 2002. LNCS, vol. 2332, pp. 83-107. Springer, Heidelberg (2002)

2. Belenkiy, M., Chase, M., Kohlweiss, M., Lysyanskaya, A.: P-signatures and noninteractive anonymous credentials. In: Canetti, R. (ed.) TCC 2008. LNCS, vol. 4948, pp. 356-374. Springer, Heidelberg (2008)

3. Bellare, M., Boldyreva, A., Desai, A., Pointcheval, D.: Key-privacy in public-key encryption. In: Boyd, C. (ed.) ASIACRYPT 2001. LNCS, vol. 2248, pp. 566-582. Springer, Heidelberg (2001)

4. Bellare, M., Rogaway, P.: Random oracles are practical: A paradigm for designing efficient protocols. In: ACM CCS 1993, pp. 62-73 (1993)

5. Boldyreva, A., Fischlin, M., Palacio, A., Warinschi, B.: A closer look at PKI: Security and efficiency. In: Okamoto, T., Wang, X. (eds.) PKC 2007. LNCS, vol. 4450, pp. 458-475. Springer, Heidelberg (2007)

6. Boneh, D., Boyen, X.: Short signatures without random oracles. In: Cachin, C., Camenisch, J.L. (eds.) EUROCRYPT 2004. LNCS, vol. 3027, pp. 56-73. Springer, Heidelberg (2004)

7. Boneh, D., Boyen, X., Shacham, H.: Short group signatures. In: Franklin, M. (ed.) CRYPTO 2004. LNCS, vol. 3152, pp. 41-55. Springer, Heidelberg (2004)

8. Boneh, D., Franklin, M.: Identity based encryption from the Weil pairing. SIAM J. of Computing 32(3), 586-615 (2003); Extended abstract in Crypto 2001, LNCS, vol. 2139, pp. 213-229 (2001)

9. Boyen, X., Delerablée, C.: Expressive subgroup signatures. In: Ostrovsky, R., De Prisco, R., Visconti, I. (eds.) SCN 2008. LNCS, vol. 5229, pp. 185-200. Springer, Heidelberg (2008) 
10. Boyen, X., Waters, B.: Full-domain subgroup hiding and constant-size group signatures. In: Okamoto, T., Wang, X. (eds.) PKC 2007. LNCS, vol. 4450, pp. 1-15. Springer, Heidelberg (2007)

11. Camenisch, J., Chandran, N., Shoup, V.: A public key encryption scheme secure against key dependent chosen plaintext and adaptive chosen ciphertext attacks. In: Ghilardi, S. (ed.) EUROCRYPT 2009. LNCS, vol. 5479, pp. 351-368. Springer, Heidelberg (2009)

12. Camenisch, J., Lysyanskaya, A.: A Signature Scheme with Efficient Protocols. In: Cimato, S., Galdi, C., Persiano, G. (eds.) SCN 2002. LNCS, vol. 2576, pp. 268-289. Springer, Heidelberg (2003)

13. Camenisch, J., Shoup, V.: Practical verifiable encryption and decryption of discrete logarithms. In: Boneh, D. (ed.) CRYPTO 2003. LNCS, vol. 2729, pp. 126-144. Springer, Heidelberg (2003)

14. Canetti, R., Goldreich, O., Halevi, S.: The random oracle methodology, revisited. Journal of the ACM 51(4), 557-594 (2004)

15. Canetti, R., Halevi, S., Katz, J.: Chosen-ciphertext security from identity-based encryption. In: Cachin, C., Camenisch, J.L. (eds.) EUROCRYPT 2004. LNCS, vol. 3027, pp. 207-222. Springer, Heidelberg (2004)

16. Chaum, D., van Heyst, E.: Group signatures. In: Davies, D.W. (ed.) EUROCRYPT 1991. LNCS, vol. 547, pp. 257-265. Springer, Heidelberg (1991)

17. Cramer, R., Shoup, V.: A practical public key cryptosystem provably secure against adaptive chosen ciphertext attack. In: Krawczyk, H. (ed.) CRYPTO 1998. LNCS, vol. 1462, pp. 13-25. Springer, Heidelberg (1998)

18. Cramer, R., Shoup, V.: Signature schemes based on the strong rsa assumption. In: ACM CCS 1999, pp. 46-51 (1999)

19. Delerablée, C., Pointcheval, D.: Dynamic fully anonymous short group signatures. In: Nguyên, P.Q. (ed.) VIETCRYPT 2006. LNCS, vol. 4341, pp. 193-210. Springer, Heidelberg (2006)

20. Fiat, A., Shamir, A.: How to prove yourself: Practical solutions to identification and signature problems. In: Odlyzko, A.M. (ed.) CRYPTO 1986. LNCS, vol. 263, pp. 186-194. Springer, Heidelberg (1987)

21. Fuchsbauer, G., Pointcheval, D.: Proofs on Encrypted Values in Bilinear Groups and an Application to Anonymity for Signatures. In: Shacham, H., Waters, B. (eds.) Pairing 2009. LNCS, vol. 5671, pp. 132-149. Springer, Heidelberg (2009)

22. Goldwasser, S., Tauman-Kalai, Y.: On the (In)security of the Fiat-Shamir Paradig. In: FOCS 2003, pp. 102-115 (2003)

23. Groth, J.: Simulation-sound NIZK proofs for a practical language and constant size group signatures. In: Lai, X., Chen, K. (eds.) ASIACRYPT 2006. LNCS, vol. 4284, pp. 444-459. Springer, Heidelberg (2006)

24. Groth, J.: Fully anonymous group signatures without random oracles. In: Kurosawa, K. (ed.) ASIACRYPT 2007. LNCS, vol. 4833, pp. 164-180. Springer, Heidelberg (2007)

25. Groth, J.: Homomorphic trapdoor commitments to group elements. Cryptology ePrint Archive: Report 2009/007 (2009)

26. Groth, J., Lu, S.: A non-interactive shuffle with pairing based verifiability. In: Kurosawa, K. (ed.) ASIACRYPT 2007. LNCS, vol. 4833, pp. 51-67. Springer, Heidelberg (2007)

27. Groth, J., Sahai, A.: Efficient non-interactive proof systems for bilinear groups. In: Smart, N.P. (ed.) EUROCRYPT 2008. LNCS, vol. 4965, pp. 415-432. Springer, Heidelberg (2008) 
28. Halevi, S.: A Sufficient Condition for Key-Privacy. Cryptology ePrint Archive: Report 2005/005 (2005)

29. Kiayias, A., Tsiounis, Y., Yung, M.: Group encryption. In: Kurosawa, K. (ed.) ASIACRYPT 2007. LNCS, vol. 4833, pp. 181-199. Springer, Heidelberg (2007)

30. Kiayias, A., Yung, M.: Group signatures with efficient concurrent join. In: Cramer, R. (ed.) EUROCRYPT 2005. LNCS, vol. 3494, pp. 198-214. Springer, Heidelberg (2005)

31. Kiltz, E.: Chosen-ciphertext security from tag-based encryption. In: Halevi, S., Rabin, T. (eds.) TCC 2006. LNCS, vol. 3876, pp. 581-600. Springer, Heidelberg (2006)

32. Krawczyk, H., Rabin, T.: Chameleon signatures. In: NDSS 2000 (2000)

33. Kunz-Jacques, S., Pointcheval, D.: About the security of MTI/C0 and MQV. In: De Prisco, R., Yung, M. (eds.) SCN 2006. LNCS, vol. 4116, pp. 156-172. Springer, Heidelberg (2006)

34. Naor, M., Yung, M.: Universal one-way hash functions and their cryptographic applications. In: STOC 1989, pp. 33-43 (1989)

35. Paillier, P.: Public-key cryptosystems based on composite degree residuosity classes. In: Stern, J. (ed.) EUROCRYPT 1999. LNCS, vol. 1592, pp. 223-238. Springer, Heidelberg (1999)

36. Qin, B., Wu, Q., Susilo, W., Mu, Y., Wang, Y.: Publicly Verifiable PrivacyPreserving Group Decryption. In: Moti, Y., Liu, P., Lin, D. (eds.) Inscrypt 2008. LNCS, vol. 5487, pp. 72-83. Springer, Heidelberg (2008)

37. Shacham, H.: A Cramer-Shoup encryption scheme from the linear assumption and from progressively weaker linear variants. Cryptology ePrint Archive: Report 2007/074 (2007)

38. Shoup, V.: A proposal for the ISO standard for public-key encryption (version 2.1). manuscript (2001), http://shoup.net/

\section{A Sketch of the Proof of Theorem 1}

The security proof of the certification scheme considers three kinds of forgeries in the attack game.

- Type I forgeries: are such that the fake certificate cert $_{\mathrm{pk}^{\star}}^{\star}$ contains a tuple of elements $\left(S_{1}^{\star}, S_{2}^{\star}, S_{3}^{\star}\right)$ that never appeared in outputs of certification queries.

- Type II forgeries: are such that cert pk $^{\star}$ contains a triple $\left(S_{1}^{\star}, S_{2}^{\star}, S_{3}^{\star}\right)$ that appeared in the output of some query but cert $\mathrm{pk}^{\star}$ also contains commitments $\left\{\left(C_{i, 1}^{\star}, C_{i, 2}^{\star}, C_{i, 3}^{\star}\right)\right\}_{i=1, \ldots, n}$ that do not match those in the output of that query.

- Type III forgeries: are such that $\left(S_{1}^{\star}, S_{2}^{\star}, S_{3}^{\star}\right)$ and $\left\{\left(C_{i, 1}^{\star}, C_{i, 2}^{\star}, C_{i, 3}^{\star}\right)\right\}_{i=1, \ldots, n}$ are identical in cert $_{\mathrm{pk}^{\star}}^{\star}$ and in the output of some certification query. On the other hand, the public key $\mathrm{pk}^{\star}=\left(X_{1}^{\star}, \ldots, X_{n}^{\star}\right)$ is not the one that was certified in that query.

Type I forgeries are easily seen to break the HSDH assumption whereas Type II and Type III forgeries give rise to algorithms solving the FlexDH and S2P problems, respectively. Due to space limitations, the details are deferred to the full version of the paper. 